\title{
Authentic display of a cholera toxin epitope by chimeric type 1 fimbriae: effects of insert position and host background
}

\author{
Bodil Stentebjerg-Olesen, ${ }^{1}$ Lars Pallesen, ${ }^{1} \dagger$ Lars Bogø Jensen, ${ }^{1} \ddagger$ \\ Gunna Christiansen² and Per Klemm
}

\author{
Author for correspondence: Per Klemm. Tel: +45 45 25 25 06. Fax: +4545932809. \\ e-mail:pk@im.dtu.dk
}

1 Department of

Microbiology, Building

301, Technical University

of Denmark, DK-2800

Lyngby, Denmark

2 Department of Medical Microbiology, University of Aarhus, DK-8000 Aarhus C, Denmark

\begin{abstract}
The potential of the major structural protein of type 1 fimbriae as a display system for heterologous sequences was tested. As a reporter-epitope, a heterologous sequence mimicking a neutralizing epitope of the cholera toxin B chain was inserted, in one or two copies, into four different positions in the fimA gene. This was carried out by introduction of new restriction sites by PCRmediated site-directed mutagenesis of fimA in positions predicted to correspond to optimally surface-located regions of the subunit protein. Subsequently, the synthetic cholera-toxin-encoding DNA segment was inserted. Several of the chosen positions seemed amenable even for large foreign inserts; the chimeric proteins were exposed on the bacterial surface and the cholera toxin epitope was authentically displayed, i.e. it was recognized on bacteria by specific antiserum. Display of chimeric fimbriae was tested with respect to host background in three different Escherichia coli strains, i.e. an isogenic set of K-12 strains, differing in the presence of an indigenous fim gene cluster, as well as a wild-type isolate. Immunization of rabbits with purified chimeric fimbriae resulted in serum which specifically recognized cholera toxin B chain, confirming the utility of the employed strategy.
\end{abstract}

Keywords: surface display of foreign epitopes, chimeric proteins, effect of host background, type 1 fimbriae

\section{INTRODUCTION}

Developments in biotechnology have led to a number of new strategies for designing and engineering vaccines. An attractive example is to fuse immunologically relevant sectors of an important protein antigen into a naturally occurring bacterial surface protein such as the structural proteins for flagella or fimbriae. Fimbriae are adhesive bacterial surface structures which enable bacteria to target and to colonize specific host tissues (for reviews see Duguid \& Old, 1980; Klemm \& Krogfelt, 1994; Krogfelt, 1991). These are long thread-like surface

\footnotetext{
†Present address: Department of Research and Development, Division of Microbiology, State Serum Institute, DK-2300 Copenhagen S, Denmark.

$\ddagger$ Present address: Danish Veterinary Laboratory, DK-1790, Copenhagen, Denmark.

Abbreviations : CTB, cholera toxin B chain; DICM, differential interference contrast microscopy; FITC, fluorescein isothiocyanate.
}

organelles, found in up to about 500 copies per cell. A large variety of fimbriae are known, and have, where this aspect has been studied, turned out to be completely non-toxic proteins only serving as colonization factors. Vaccines based on wild-type fimbriae have been highly successful, for example in protecting against various diarrhoea-causing enterotoxigenic Escherichia coli strains (Levine et al., 1994; Moon \& Bunn, 1993). Such results have indicated that fimbriae normally are very good immunogens both in the context of live vaccines and as purified proteins.

Type 1 fimbriae are found on the majority of E. coli strains. A single type 1 fimbria is a thin, $7 \mathrm{~nm}$ wide and approximately $1 \mu \mathrm{m}$ long, surface polymer. It consists of about 1000 subunits of a major building element, the FimA protein, stacked in a helical cylinder (Brinton, 1965). Additionally, a small percentage of minor components are also present as integral parts of the fimbriae (Krogfelt \& Klemm, 1988). It has been shown that the minor components, FimF, FimG and FimH, are involved 


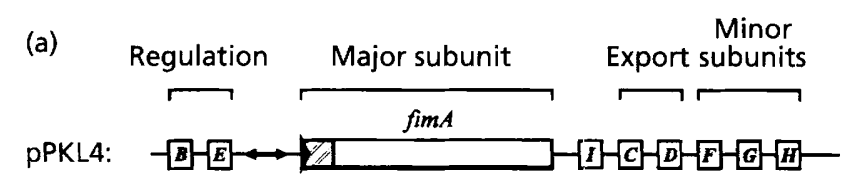

(b)

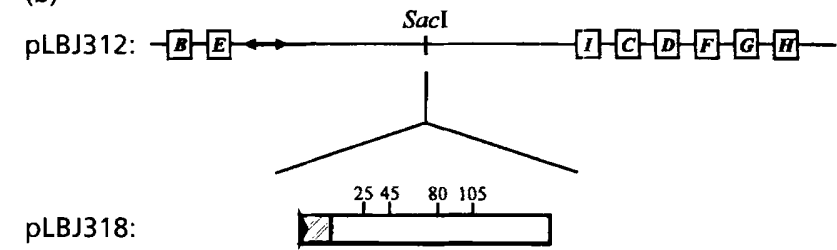

(c)

$1 \times$ Cholera:

Bgnt

AGA TCT GTT GAA GTT CCG GGT AGT CAG CAT ATC GAT AGT CAG AAA AAA

Arg Ser Val Glu Val Pro Gly Ser Gin His De Asp Ser Gla Lys Lys

BamHL/BgII

GCT GGA TCT

Ala Gly S

$2 \times$ Cholera:

BgIII

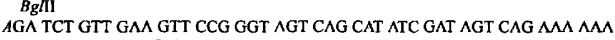
Ang Ser Val Gtu Val Pro Gly Ser Gin His the Asp Ser Gin Lys Lys

BamHU $B g$ III

GCT GGA TCT GTT GAA GTT CCG GGT AGT CAG CAT ATC GAT AGT CAG AAA Ala Gly Ser Val Glu Val Pro Gly Ser Gh His Ile Asp Ser Gin Lys BamHI/BglII

AAA GCT GGA TCT
Lys Ala Gly Ser

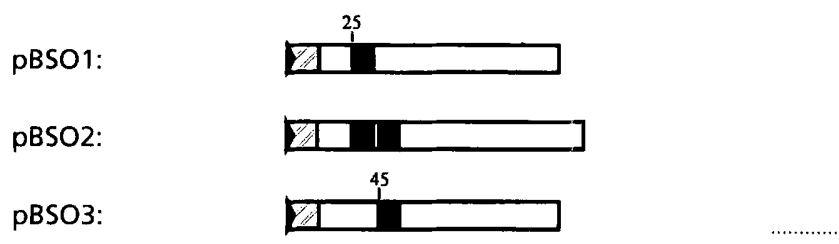

Fig. 1. Overview of plasmids (not drawn to scale). (a) fim gene cluster as present in plasmid pPKL4. Below is the replacement vector containing a Sacl site into which cassettes of engineered fimA genes were inserted. (b) Expanded section of the fimA gene showing the engineering of the fim gene cluster to contain chimeric fimA with heterologous sequences of CTB. Hatched and black boxes indicate signal-peptide- and CTBencoding sectors, respectively. Numbers above the gene boxes indicate insert positions. (c) Sequence of the synthetic DNA segment mimicking a sector of CTB. in fimbrial-length regulation (Klemm \& Christiansen, 1987; Russell \& Orndorf, 1992). The FimH protein has been shown to be the actual receptor-binding molecule which recognizes D-mannose-containing structures (Krogfelt et al., 1990). The FimF and FimG components seem to be required for integration of the FimH adhesin into the fimbriae. The minor components are per se not necessary for synthesis of the fimbriae, since recombinant bacteria entirely missing the corresponding genes are able to express pure FimA fimbriae (Klemm \& Christiansen, 1987; Krogfelt \& Klemm, 1988). However, such organelles are devoid of adherence activity.

Type 1 fimbriae are encoded by the fim gene cluster. This encompasses nine genes contained within a $9.5 \mathrm{~kb}$ DNA segment (Fig. 1). We have studied the fim genes responsible for type 1 fimbriation of E. coli K-12 strain PC.31 for a number of years and have reached a detailed understanding of the biosynthesis of these adhesive organelles. The structural components of type 1 fimbriae are produced as precursors having an N-terminal signal sequence. This element is subsequently removed during export across the inner membrane. All evidence suggests that this translocation is dependent on the normal type II $E$. coli export system, viz. SecA, SecB, etc. (Dodd \& Eisenstein, 1984; Pugsley, 1993). However, further export to the cell exterior, i.e. from the periplasm and across the outer membrane, is dependent on a fimbriaespecific export and assembly system constituted by the FimC and FimD proteins. FimC is a periplasmic located chaperone that binds to nascently translocated organelle components and prevents them from making incorrect interactions that cause aggregation and proteolytic degradation (Jones et al., 1993; Klemm, 1992; Pugsley, 1993). FimD, a so-called usher, is found in the outer membrane and is involved in organized polymerization of the structural components into fimbrial organelles (Hultgren et al., 1993; Klemm \& Christiansen, 1990; Pugsley, 1993).

The biogenesis machinery of type 1 fimbriae has been shown to be quite tolerant in accepting similar but heterologous structural components and still assemble such into fimbriae. Thus, components from F1C fimbriae exhibiting as little as $34 \%$ identity with their equivalents in type 1 fimbriae are readily integrated into type 1 fimbriae resulting in hybrid organelles (Klemm et al., 1994). This kind of information suggested that type 1 fimbriae could be used as carriers of heterologous sequences representing foreign epitopes. Such fimbriae consist of chimeric FimA proteins resulting from inframe fusion of the heterologous sequences into selected positions in the fim $A$ gene. If insert positions are chosen which do not interfere with the bioassembly of the organelles, normal fimbriae, in which every subunit will carry a foreign peptide segment will result. There are several advantages of such an approach, one being the large amounts of major subunit protein and thereby foreign antigen produced, i.e. a single fimbriated cell has as many as half a million copies of FimA on its surface. 
Another advantage is that type 1 fimbriae are excellent immunogens, which could boost the immune response against a passenger epitope.

In a previous study we inserted foreign sequences into FimA in positions corresponding to available unique restriction sites in the fimA sequence (Hedegaard \& Klemm, 1989). Questions remaining open from this analysis included the best localities and permissive size of the inserts, and significantly, whether the foreign sequences were authentically displayed in the context of chimeric FimA proteins, and could evoke an immune response directed against the parental protein. Here we try to answer these questions.

\section{METHODS}

Bacterial strains and growth conditions. A list of E. coli strains used in this study is provided in Table 1. E. coli strain MS7, a $\Delta$ fim-derivative of PC31, was made essentially following the procedure of Schembri et al. (1996). In short, an $n p t$ cassette flanked by DNA segments containing the upstream and downstream regions of the fim gene cluster, respectively, was used for homologous double crossover recombination. Cells were grown on solid medium or liquid broth supplemented with the appropriate antibiotics.

Plasmids. Plasmids used in this study are listed in Table 1. Plasmid pLPA41 was made by inserting a 636 bp PCR fragment containing the fim $A$ gene and the adjacent regions $70 \mathrm{bp}$ upstream and $20 \mathrm{bp}$ downstream and flanked by two SacI sites into the SacI site of plasmid pUC18. The insert was positioned downstream and in an expression-compatible orientation to the lac promoter residing on the vector part of the plasmid. Plasmids pLPA110, pLPA111, pLPA112 and pLPA119 were made by joining two individual PCR fragments in each case (see Fig. 2) at their BglII sites and inserting the resulting fragment into the Sacl site of plasmid pUC18. Plasmids pLPA113, pLPA114, pLPA115 and pLPA125 were made by inserting a 51 bp synthetic dsDNA segment encoding amino acids $50-64$ of the $B$ subunit of cholera toxin and containing a $B g l \mathrm{II}$ overhang at one end and a BamHI overhang at the other into the BglII site of plasmids pLPA110, pLPA111, pLPA112 and pLPA119, respectively (cf. Figs 1 and 2). Plasmids pLPA123, pLPA124 and pLPA126 were made by introducing a second cholera-epitope-encoding segment into the BglII sites of plasmids pLPA113, pLPA114 and pLPA125, respectively. Plasmid pLBJ312 was made as depicted in Fig. 3.

Prediction of surface-located positions in FimA. Positions with maximum probability of surface location were chosen according to concomitant occurrence of predicted hydrophilicity maxima and $\beta$-turns as described by Hedegaard \& Klemm (1989).

DNA techniques. Isolation of plasmid DNA was carried out by the method of Birnboim \& Doly (1979). Restriction endonucleases were used according to the manufacturer's specifications (New England Biolabs). DNA sequencing was by the dideoxy chain-termination technique (Sanger et al., 1977) using a Sequenase version 2.0 kit from USB. Oligonucleotides were made at the department's core facilities.

PCR methodology. PCR were performed on a Perkin Elmer Cetus DNA Thermal Cycler 480. Reactions were set up as $100 \mu \mathrm{l}$ volumes containing $200 \mu \mathrm{M}$ each of dATP, dCTP,
dGTP and dTTP, $0 \cdot 2-1 \cdot 0 \mu \mathrm{M}$ of each of the two primers, $2 \mathrm{mM} \mathrm{MgCl}, 10 \mathrm{mM}$ Tris/ $\mathrm{HCl}(\mathrm{pH} \mathrm{8.3)}, 50 \mathrm{mM} \mathrm{KCl}, 2.5$ units of AmpliTaq DNA polymerase and $0 \cdot 1-0 \cdot 2 \mu \mathrm{g}$ plasmid template. The reactions were run for 25-30 cycles each consisting of $1 \mathrm{~min}$ at $94^{\circ} \mathrm{C}, 1 \mathrm{~min}$ at $40^{\circ} \mathrm{C}$, and $1 \mathrm{~min}$ at $72{ }^{\circ} \mathrm{C}$. The following primers, partially based on the fimA sequence (Klemm, 1984) were used for introduction of flanking SacI sites and an internal BglII site: P1: 5'-GTCGAGCTCGTTTACAAA-3' ; P2: 5'-CAAGATCTGTTGATCAAA- $3^{\prime}$; P3: 5'-ACAGATCTTGCATCAACT-3'; P4: 5'-AAAGATCTACCAGTTCTG-3'; P5 : 5'-GTAGATCTTTCCTGTGCC3'; P6: 5'-CGAGATCTACCAACGTTC-3'; P7: 5'-GTAGATCTCGCATCAATC-3' ; P8: 5' -ACAGATCTGGTGCTGCGC-3'; P9: 5'-CCAGATCTGTCCAGGATC-3'; P10: 5'AATGAGCTCCCTGAACCT-3'. PCR fragments were made, using primers $\mathrm{P} 1-\mathrm{P} 10$, as shown in Fig. 2 employing plasmid pLPA41 as template.

Haemagglutination. The capacity of bacteria to express a Dmannose-binding phenotype was assayed by their ability to agglutinate guinea-pig erythrocytes on glass slides. Aliquots of liquid bacterial cultures at $\mathrm{OD}_{550} 10.0$ and $5 \%$ erythrocytes were mixed and the time until agglutination occurred was measured.

Antisera. Rabbit anti-type 1 fimbrial serum raised against purified type 1 fimbriae has previously been described (Krogfelt \& Klemm, 1988). Hyperimmune serum against cholera toxin $\mathrm{B}$ chain (CTB) was obtained by immunizing a rabbit with CTB (Sigma). Aliquots of $20 \mu$ l of a solution of $1 \mathrm{mg} \mathrm{CTB} \mathrm{ml}{ }^{-1}$ in Freund's incomplete adjuvant were given intravenously every fifth day over a 7 week period, after which the animal was bled. Fluorescein isothiocyanate (FITC) conjugated anti-rabbit serum was supplemented by Dako, Denmark.

Western blot (immunoblot). Samples were subjected to sodium dodecyl sulfate-polyacrylamide $(15 \%)$ gel electrophoresis and transferred to PVDF microporous membrane filters using a semi-dry blotting apparatus. Filters were processed as previously described (Hedegaard \& Klemm, 1989; Krogfelt \& Klemm, 1988). Serum raised against either wild-type or chimeric fimbriae or CTB, respectively, was used as the primary serum and peroxidase-conjugated anti-rabbit serum was used as the secondary serum.

Fixation of bacterial cells and fluorescence labelling. Cells from overnight cultures were harvested and washed gently in PBS $(0 \cdot 145 \mathrm{M} \mathrm{NaCl}, 0 \cdot 15 \mathrm{M}$ sodium phosphate). Cells were fixed by mixing $250 \mu \mathrm{l}$ of cells with $750 \mu \mathrm{l}$ of a $4 \%(\mathrm{w} / \mathrm{v})$ solution of paraformaldehyde in PBS. This mixture was incubated on ice for $20 \mathrm{~min}$. To remove the fixative, cells were washed twice in PBS. Samples of $20 \mu \mathrm{l}$ were placed on a poly $\mathrm{L}$-lysine coated slide and air dried. After washing in PBS, $16 \mu \mathrm{l}$ of a 1:100 dilution (anti-fimbriae) or 1:250 dilution (antiCTB) of the primary antiserum was placed on top of each sample and left in a moist incubation chamber for $1 \mathrm{~h}$. The slides were washed three times in PBS, and $16 \mu \mathrm{l}$ of a $1: 25$ dilution of FITC conjugated secondary antiserum was added. After $2 \mathrm{~h}$ in the dark the slides were washed three times in PBS and a drop of Citifluor (Citifluor) was placed on top of each sample.

Microscopy and image analysis. Differential interference contrast microscopy (DICM) and image analysis was carried out as described by Pallesen et al. (1995).

Electron microscopy. Electron microscopy and immunoelectron microscopy were carried out essentially as described previously (Klemm et al., 1994; Pallesen et al., 1995). In short 
a $10 \mu \mathrm{l}$ aliquot of bacterial suspension was placed on a carboncoated, glow discharged grid for $30 \mathrm{~s}$. Grids were washed in two drops PBS, dehydrated in increasing concentrations of ethanol, blotted dry, and shadowed with tungsten. For immunoelectron microscopy, a mixture of anti-CTB rabbit serum (diluted $1: 100$ ) and a monoclonal mouse anti-FimA serum (diluted 1:5) was used as the primary antiserum, and anti-rabbit and anti-mouse serum conjugated with colloidal gold particles, of $10 \mathrm{~nm}$ and $5 \mathrm{~nm}$ diameter, respectively (BioCell), were used as the secondary antibody. The monoclonal anti-FimA serum was a generous gift from Karen Krogfelt, State Serum Institute, Denmark.

Immunization with fimbriae. Fimbriae were purified essentially as previously described (Hedegaard \& Klemm, 1989), the only change being that precipitation of fimbriae from the crude extract was performed in $20 \%(\mathrm{w} / \mathrm{v})$ ammonium sulfate. Purified fimbriae were used to immunize rabbits. Two animals were immunized with purified chimeric (FimA-CTB) firmbriae, and one animal was immunized with wild-type type 1 fimbriae (control), respectively. Three doses of a suspension of $0.1 \mathrm{ml} 0.5 \mathrm{mg}$ fimbriae $\mathrm{ml}^{-1}$ mixed with equal parts of Freund's incomplete adjuvant were administered subcutaneously at 2 week intervals. Blood was collected from animals before immunization and after 3,6 and 9 weeks.

\section{RESULTS}

\section{Research strategy}

In a preliminary study (Hedegaard \& Klemm, 1989) heterologous DNA segments, encoding consensus sequences for restriction enzymes or, in a few cases, mimicking foreign epitopes, were inserted into naturally available restriction sites in the fim $A$ gene which were unique in the context of all or large parts of the fim gene cluster. Such an approach only permits insertion into a few positions which might not be optimal for harbouring heterologous sequences. The present work relies on a different strategy, viz. introduction of restriction sites by PCR technology. Such an approach offers the possibility to engineer any position of choice in the fim $A$ gene to contain unique restriction sites which in turn can be used for insertion of heterologous sequences representing foreign epitopes. In this work, initial genetic manipulations of the fimA gene were carried out on plasmid vectors that uniquely contained this gene. To avoid cumbersome binary plasmid systems, all manipulated fim $A$ chimeras were subsequently moved into the context of the total fim gene cluster on a single plasmid.

\section{Engineering new restriction sites into fimA}

Based on algorithms for prediction of such parameters as hydrophilicity and secondary structure, five positions, corresponding to $25,45,60,80$ and 105 in the mature FimA protein were predicted to have maximum probability of surface-exposure in the protein. One of these, position 60 , is proximal to a cysteine residue known to be involved in the intramolecular $S_{21}-S_{61}$ bridge (McMichael \& Ou, 1979). To avoid interfering with this structural feature we decided to concentrate on the four other positions, viz. 25, 45, 80 and 105, as insertion sites for heterologous sequences. To facilitate later manipulations the fimA gene was subcloned into the SacI site of the pUC18 vector by PCR technology (cf. Methods) resulting in plasmid pLPA41. Subsequently, BgllI sites were introduced into the fim $A$ gene in locations corresponding to the previously mentioned positions in the mature FimA protein. This was carried out by sitedirected mutagenesis employing synthetic oligonucleotides and PCR amplification resulting in plasmids pLPA110, pLPA119, pLPA111 and pLPA112 (Fig. 2). The introduced $B g l$ II sites did not result in any change in the number of amino acids, but did cause a few codon changes. Sequence analysis of the entire modified fimA genes in plasmids pLPA110, pLPA119, pLPA111 and pLPA112 confirmed that no other changes had occurred.

\section{Engineering heterologous DNA sequences encoding a cholera toxin epitope into four sites in fimA}

As a relevant heterologous reporter-epitope we have chosen a well-characterized region of the CTB. This segment consists of residues 50-64 of CTB and has previously been shown to elicit antibodies that bind to and neutralize cholera toxin (Jacob et al., 1983). Residues 50-64 comprise a conformational loop on the surface of CTB that is entirely conserved between CTB and the closely related heat-labile toxin of E. coli (Merrit et al., 1994; Shoham et al., 1995). This loop is directly involved in receptor-binding of the toxin to epithelial cells (Merrit et al., 1994). A synthetic DNA segment encoding the cholera epitope was made by annealing two complementary 51 bp oligonucleotides, which were designed, after annealing, to result in a dsDNA segment with a $B g l I I$ overhang in one end, and a $B a m H I$ overhang in the other. A single copy of the epitope-encoding segment was inserted into the $B g l I I$ sites in the fim $A$ gene in plasmids pLPA110, pLPA119, pLPA111 and pLPA112, respectively, resulting in plasmids pPLA113, pLPA125, pLPA114 and pLPA115 (Fig. 2). Insertion of the cholera-toxin-encoding oligonucleotide resulted in regeneration of a $B g l I I$ site at only one end of the insert. This feature was used to identify plasmids with correct orientation of the insert. Furthermore, this feature also permitted creation of a number of chimeras containing tandem repeats of the cholera toxin insert, i.e. plasmids pLPA123, pLPA126 and pLPA124 (Table 1; Fig. 2). Restriction enzyme and DNA sequence analysis of the chimeric plasmids, harbouring either a single or double cholera insert, confirmed the orientation and conservation of the reading frame in the chimeric fimAcholera gene.

\section{Construction of single plasmid systems containing fim gene clusters with chimeric versions of fimA}

To create a cassette into which chimeric versions of the fim $A$ gene could be easily inserted into a background of the rest of the fim genes, the fim $A$ gene was replaced by a unique $S a c I$ restriction site resulting in plasmid pLBJ312 (Fig. 3). A fim host containing this plasmid is 


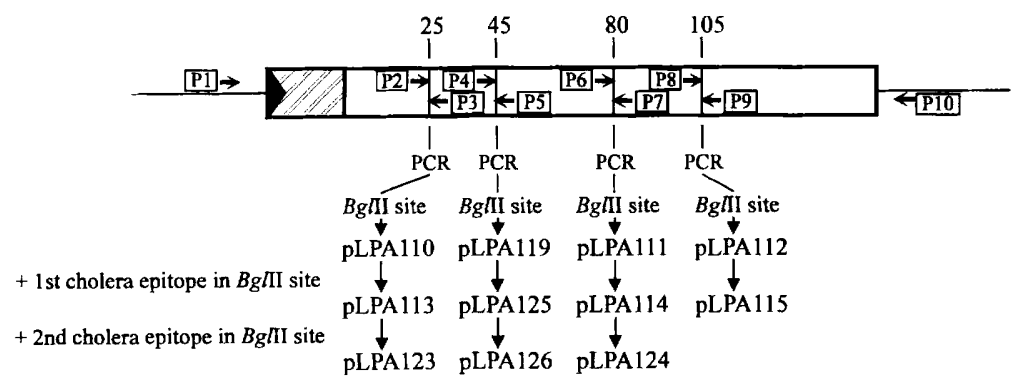

Fig. 2. Flow diagram showing the pedigree of the engineering of the fimA gene to contain Bg/ll handles, and segments of CTB. Numbers correspond to manipulated positions; numbers in boxes refer to the employed primers (cf. Methods).

unable to produce fimbriae. To test the replacement system a SacI fragment from plasmid pLPA41, containing the wild-type version of the fim $A$ gene, was introduced into the Sacl site of pLBJ312 resulting in pLBJ318 (Fig. 1). Host cells that harboured this plasmid produced type 1 fimbriae which in quality and quantity were indistinguishable from those produced by hosts containing a control plasmid (pPKL4) that harboured the non-engineered fim gene cluster (data not shown). Since all chimeric fimA genes in plasmids pLPA113pLPA115 and pLPA123-pLPA126 were designed such that they were flanked by SacI sites, all of these cassettes were subsequently inserted into the unique SacI site of pLBJ312, resulting in plasmids pBSO1-pBSO 7, respectively (Table 1, Fig. 1).

\section{Expression of chimeric FimA and chimeric type 1 fimbriae}

The ability of E. coli hosts, which contained plasmids encoding chimeric fim $A$ genes, to express fimbriae was assayed by such criteria as immunoblotting, haemagglutination, electron and immunofluorescence microscopy. Immunoblotting employing anti-fimbrial serum of bacterial hosts that harboured the recombinant plasmids in most cases revealed the presence of chimeric proteins with the expected molecular masses (Fig. 4). The most notable examples were pBSO1 and pBSO2, encoding fimA genes with one or two heterologous inserts in position 25, respectively, which resulted in protein levels comparable to non-chimeric controls (pLBJ318). At the other end of the spectrum were strains from which very little or no recombinant protein could be observed, e.g. hosts containing plasmid pBSO7 with a single insert in position 105 . The haemagglutination titres of host cells containing chimeric fim $\mathrm{A}$-cholera genes were generally lower than those of comparable wild-type fim $A$ controls (Table 2).

\section{Immunological detection of the cholera toxin epitope in the context of chimeric type 1 fimbriae}

In Western blots of total lysates of host cells harbouring plasmids pBSO1-pBSO7 chimeric FimA-CTB fimbriae were not recognized by specific anti-CTB serum. In the light of the fact that residues 50-64 form a conformational loop it is perhaps not surprising that this feature is not correctly re-folded in chimeric FimA-CTB proteins after extensive denaturation. It should be noted that this loop is indeed re-folded in the context of the parental protein, CTB (see later), however, the molecular environment of the loop present in the chimeras or in the parental molecular background is obviously quite different. Therefore, we investigated if the heterologous segments were authentically displayed directly on the bacteria. This was studied by CCD (chargecoupled device) microscopy in combination with fluorescent antibody methodology. By this technique E. coli hosts expressing chimeric FimA-CTB fimbriae were specifically recognized by serum raised against cholera toxin B subunit. Fig. 5 shows two representative examples of surface display of chimeric FimA proteins, harbouring a single and a tandem repeat of the heterologous sequence, respectively. In both cases the chimeric proteins are recognized by insert-specific antiserum. This demonstrates that the foreign epitopes are exposed on the surface of the host cells and, notably, in a conformation which mimics the natural conformation of the epitope in the context of the cholera toxin. We also investigated surface-localization and organelle assembly of the chimeric proteins by electron and immunoelectron microscopy (Fig. 6). The data indicate that the chimeric proteins were specifically recognized on the surface of bacterial hosts by anti-CTB serum. The chimeric proteins were also, at least to some extent, assembled into fimbriae (Fig. 6c, e and g).

\section{Expression of chimeric fimbriae is dependent on the host background}

In the present study we have investigated the expression of chimeric FimA and fimbriae in three different strains. One, MS7, is $\Delta$ fim, permitting us to investigate the ability of the introduced plasmids to confer fimbrial synthesis without interference from a chromosomally located fim gene cluster. The isogenic $\mathrm{fim}^{+}$parent strain PC31, was used to provide information about the influence of a resident fim gene cluster on the plasmidencoded systems. Additionally, we employed a commensal isolate, DSM 6601, which is also $\mathrm{fm}^{+}$(Blum et al., 1995). Comparing the MS7 and PC31 strains there seemed to be a tendency that a resident fim gene cluster provided a better background for expression of chimeric FimA proteins and fimbriae. For example, in the case of plasmid pBSO2 a high level of chimeric protein was seen in immunoblotting in a PC31 background, whereas 
Table 1. Bacterial strains and plasmids

\begin{tabular}{|c|c|c|}
\hline Strain/plasmid & Relevant genotype & Reference or construction \\
\hline PC31 & gal, tonA, phx $, \arg F, r e l$ & Klemm et al. $(1985)$ \\
\hline MS7 & $\mathrm{PC} 31 \Delta \mathrm{fim}$ & $\begin{array}{l}\text { fim gene cluster replaced by a } n p t \text { casette (cf. } \\
\text { Methods) }\end{array}$ \\
\hline DSM 6601 & $f m^{+}$ & Blum et al. (1995) \\
\hline pPKL4 & All fim genes & Klemm et al. (1985) \\
\hline pLBJ312 & $f i m A$ & $\begin{array}{l}\text { Contains a fim gene cluster in which the fimA gene } \\
\text { is replaced by a Sacl site }\end{array}$ \\
\hline pLBJ318 & All fim genes & fimA gene inserted in the $S a c \mathrm{I}$ site of pLBJ 312 \\
\hline pLPA41 & $f i m A^{+}$ & $\begin{array}{l}\text { PCR fragment containing the fimA gene inserted in } \\
\text { the } S a c \text { I site of pUC18 }\end{array}$ \\
\hline pLPA110 & fimA-BglII $(25)$ & $\begin{array}{l}\text { BglII linker inserted in-frame in a position } \\
\text { corresponding to aa } 25 \text { in the fimA gene of pLPA } 41\end{array}$ \\
\hline pLPA119 & fim $A-B g / I I(45)$ & $\begin{array}{l}\text { BglII linker inserted in-frame in a position } \\
\text { corresponding to aa } 45 \text { in the fimA gene of pLPA } 41\end{array}$ \\
\hline pLPA111 & fim $A-B g l \mathrm{II}(80)$ & $\begin{array}{l}B g l \text { II linker inserted in-frame in a position } \\
\text { corresponding to aa } 80 \text { in the fim } A \text { gene of pLPA } 41\end{array}$ \\
\hline pLPA112 & fim $A-B g l \mathrm{II}(105)$ & $\begin{array}{l}\text { BglII linker inserted in-frame in a position } \\
\text { corresponding to aa } 105 \text { in the fimA gene of } \\
\text { pLPA41 }\end{array}$ \\
\hline pLPA113 & fimA-cholera (25) & $\begin{array}{l}\text { DNA segment encoding } 15 \text { aa of CTB inserted in the } \\
\text { BglII site of pLPA110 }\end{array}$ \\
\hline pLPA125 & fimA-cholera (45) & $\begin{array}{l}\text { DNA segment encoding } 15 \text { aa of CTB inserted in the } \\
\text { Bglll site of pLPA119 }\end{array}$ \\
\hline pLPA114 & fimA-cholera $(80)$ & $\begin{array}{l}\text { DNA segment encoding } 15 \text { a of CTB inserted in the } \\
\text { BglII site of pLPA111 }\end{array}$ \\
\hline pLPA115 & fimA-cholera $(105)$ & $\begin{array}{l}\text { DNA segment encoding } 15 \text { aa of CTB inserted in the } \\
\text { BgllI site of pLPA112 }\end{array}$ \\
\hline pLPA123 & fimA-2 $\times$ cholera $(25)$ & $\begin{array}{l}\text { pLPA } 110 \text { containing a tandem CTB segment inserted } \\
\text { in the BgllI site }\end{array}$ \\
\hline pLPA126 & fimA-2 $\times$ cholera $(45)$ & $\begin{array}{l}\text { pLPA119 containing a tandem CTB segment inserted } \\
\text { in the BglII site }\end{array}$ \\
\hline pLPA124 & fimA-2 $\times$ cholera $(80)$ & $\begin{array}{l}\text { pLPA } 111 \text { containing a tandem CTB segment inserted } \\
\text { in the BglII site }\end{array}$ \\
\hline pBSO1 & fimA-cholera (25) & $\begin{array}{l}\text { SacI fragment containing the chimeric fimA-cholera } \\
\text { gene from pLPA113 inserted in the SacI site of } \\
\text { pLBJ312 }\end{array}$ \\
\hline pBSO2 & fimA-2 $\times$ cholera $(25)$ & $\begin{array}{l}\text { Sacl fragment containing the chimeric fimA-cholera } \\
\text { gene from pLPA123 inserted in the SacI site of } \\
\text { pLBJ312 }\end{array}$ \\
\hline pBSO3 & fimA-cholera (45) & $\begin{array}{l}\text { SacI fragment containing the chimeric fimA-cholera } \\
\text { gene from pLPA125 inserted in the SacI site of } \\
\text { pLBJ } 312\end{array}$ \\
\hline pBSO4 & fimA-2 $\times$ cholera $(45)$ & $\begin{array}{l}\text { SacI fragment containing the chimeric fimA-cholera } \\
\text { gene from pLPA126 inserted in the SacI site of } \\
\text { pLBJ312 }\end{array}$ \\
\hline pBSO5 & fimA-cholera (80) & $\begin{array}{l}\text { Sacl fragment containing the chimeric fimA-cholera } \\
\text { gene from pLPA114 inserted in the SacI site of } \\
\text { pLBJ312 }\end{array}$ \\
\hline pBSO6 & fimA-2 $\times$ cholera $(80)$ & $\begin{array}{l}\text { Sacl fragment containing the chimeric fimA-cholera } \\
\text { gene from pLPA124 inserted in the SacI site of } \\
\text { pLBJ } 312\end{array}$ \\
\hline pBSO7 & fimA-cholera (105) & $\begin{array}{l}\text { SacI fragment containing the chimeric fimA-cholera } \\
\text { gene from pLPA115 inserted in the SacI site of } \\
\text { pLBJ } 312\end{array}$ \\
\hline
\end{tabular}

* Numbers in brackets indicate insert positions. 


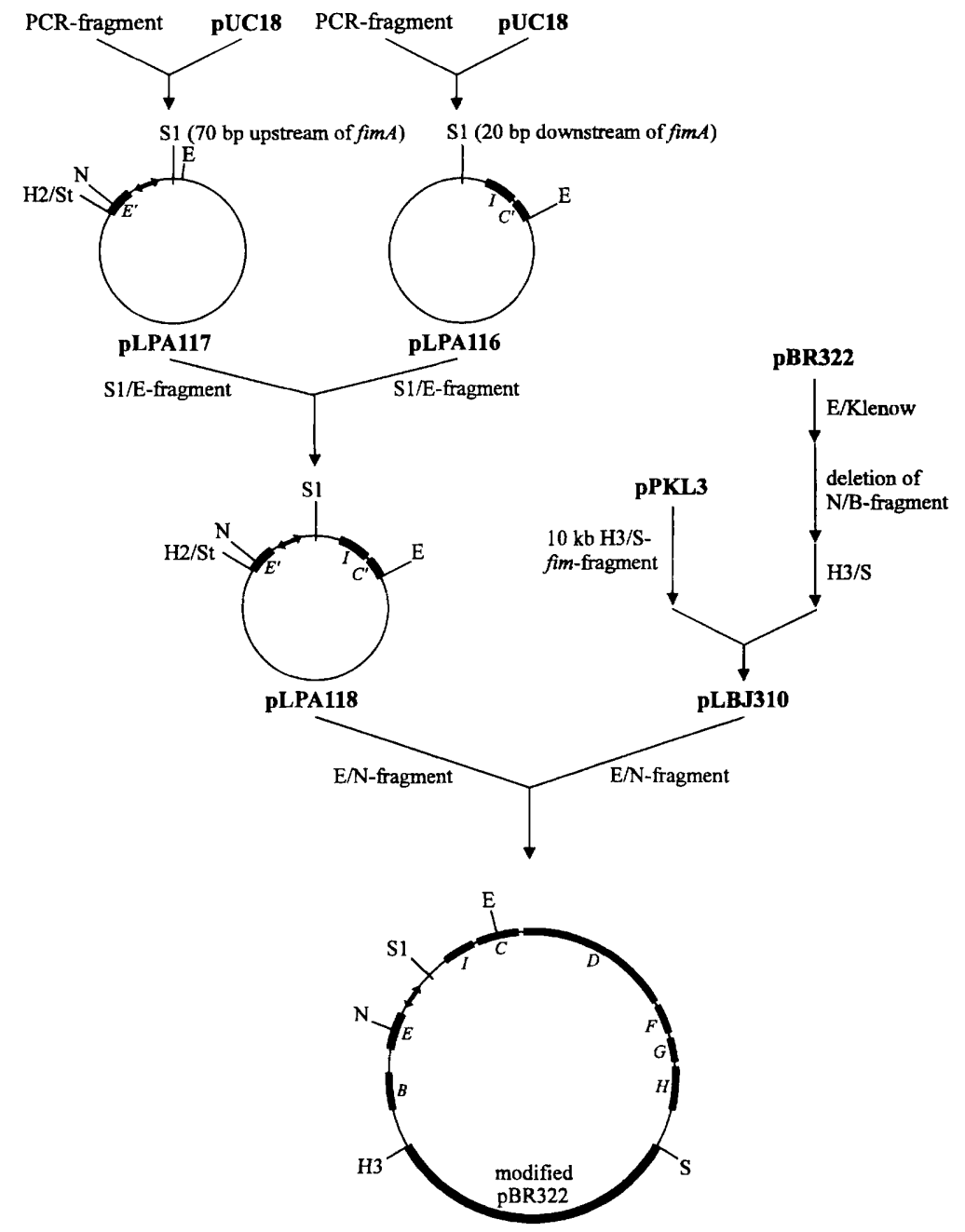

pLBJ312
Fig. 3. Construction of the replacement vector, pLBJ312, harbouring a unique $\mathrm{SaCl}$ site for integration of engineered $\operatorname{fimA}$ genes into the fim gene cluster. Relevant restriction sites are indicated: $\mathrm{B}, \mathrm{BsaBl} ; \mathrm{H} 2$ HindII; H3, HindIII; E, ECoRI; N, Nrul; S, Sall; S1, Sacl; St, Stul.

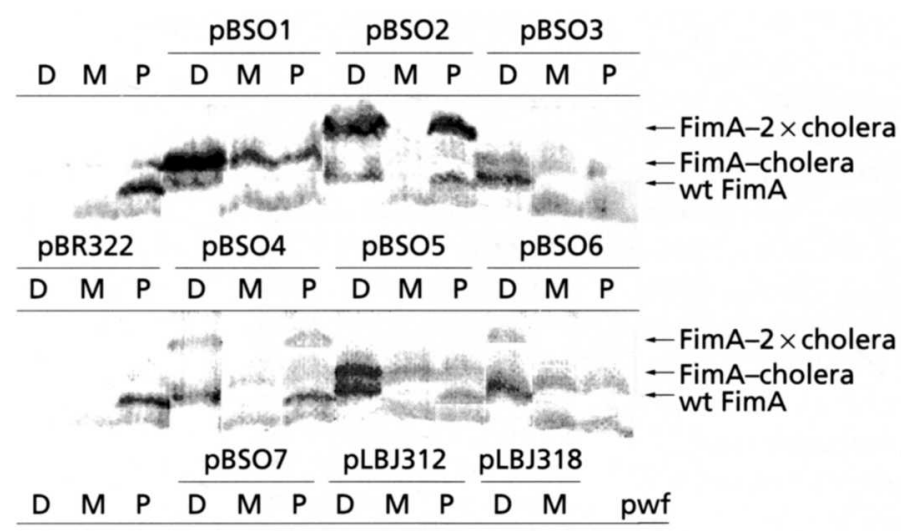

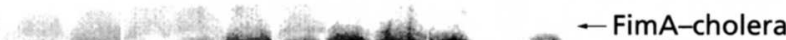
- wt FimA
Fig. 4. Production of fimbriae from recombinant strains assayed by Western blot of total cell lysates of $E$. coli DSM 6601 (D), MS7 (M) and PC31 (P) hosts containing plasmid pBSO1-pBSO7, pLBJ312, pLBJ318 and PBR322; 'pwf' indicates purified wildtype fimbriae. Rabbit anti-fimbrial serum was used as primary serum and peroxidasecoupled anti-rabbit serum was employed as secondary serum. none was seen in the case of MS7 (Fig. 4). Meanwhile, judging from all the criteria we have used, strain DSM 6601 is apparently the best host for expression of chimeric FimA proteins. With the exception of plasmid pBSO7 all chimeric FimA proteins were expressed and exposed on the surface of DSM 6601. 
Table 2. Fimbriation level of various strains assayed by haemagglutination

Values are time (s) before reaction occurred (means of three measurements). Subscript numbers indicate the corresponding positions in the mature FimA protein after which the heterologous sequence was inserted.

\begin{tabular}{|llrrr|}
\hline Plasmid & \multirow{2}{*}{$\begin{array}{c}\text { Relevant plasmid } \\
\text { genotype }\end{array}$} & \multicolumn{3}{c|}{ Strain } \\
\cline { 3 - 5 } & & DSM 6601 & PC31 & MS7 \\
\hline None & & $>300$ & 16 & $>300$ \\
pBR322 & & $>300$ & 17 & $>300$ \\
pLBJ318 & All fim genes & 6 & 5 & 5 \\
pLBJ312 & fim $A$ & 8 & 5 & $>300$ \\
pBSO1 & fim $A-1$ cholera & & \\
pBSO2 & fim $A-2$ cholera & 20 & 65 & 50 \\
pBSO3 & fim $A-1$ cholera & 19 & 43 & $>300$ \\
pBSO4 & fim $A-2$ cholera & 17 & 33 & 60 \\
pBSO5 & fim $A-1$ choler & 22 & 47 & $>300$ \\
pBSO6 & fim $A-2$ cholera & 9 & 12 & 15 \\
pBSO7 & fim $A-1$ cholera & 15 & 50 & 140 \\
\hline
\end{tabular}

\section{Immunogenicity of chimeric fimbriae}

Chimeric FimA-CTB fimbriae were purified from hosts containing plasmid pBSO1 (a single CTB segment in position 25), and used to immunize rabbits. As control one rabbit was immunized with wild-type fimbriae. Serum from animals immunized with the chimeric fimbriae was specifically able to recognize CTB in immunoblots, whereas serum from the control, immunized with wild-type fimbriae, was not (Fig. 7).

\section{DISCUSSION}

In this study we have expressed the sequence of an important neutralizing epitope of CTB in localities of the FimA protein predicted to be surface-located. Our data demonstrate that this was indeed the case: the inserted cholera toxin epitope was presented in an antigenic configuration mimicking that in the toxin, and was exposed on the surface of the bacterial hosts. The C.TB segment was recognized in the context of chimeric FimA proteins by two independent methods, i.e. fluorescence microscopy and immunoelectron microscopy, on the surface of bacterial hosts (Figs 5 and 6). Also, the chimeric proteins were to some extent polymerized into fimbriae (Fig. 6). In one of the constructs, harbouring the heterologous insert in position 80 , the chimeric protein was recognized by antiCTB serum but not by anti-FimA monoclonal antibodies (mAbs) (Fig. 6c, e and g). However, the mAbs were able to recognize wild-type FimA as well as chimeric FimA with an insert in position 25 (Fig. 6a and h). Consequently, the CTB insert in position 80 must either destroy or shield the specific epitope recognized by the mAbs. The CTB epitope present in the context of chimeric fimbriae also elicited a specific immune response in rabbits (Fig. 7).
Of the four positions in FimA $(25,45,80$ and 105) that we have tested for display of the reporter epitope, only position 105 proved incompatible with the approach, whereas inserts in the other positions resulted in production of chimeric FimA. Large heterologous inserts seemed to be tolerated in FimA, provided the insert position was amenable to engineering. Insertion of 34 foreign amino acids (a tandem repeat of the CTB segment) in positions 25 and 45 of the FimA sequence, respectively, in both cases resulted in synthesis and surface-presentation of the chimeric proteins. Several factors can influence the tolerance for heterologous inserts in the FimA protein. Insertion into domains critical for the structural integrity of the protein and those involved in subunit-subunit interaction, and in interactions with the fimbrial biogenesis machinery, i.e. the FimC chaperone and the FimD usher protein, would probably not be tolerated. However, since the threedimensional structure of FimA is unknown, a strategy based on computer algorithms for prediction of surfaceexposed sectors for insertion of heterologous inserts seems to work reasonably well, judging from the fact that three out of the four positions were in fact compatible with the strategy.

The presence of an indigenous wild-type fim gene cluster apparently had a positive effect on the production and surface exposure of the chimeric proteins. The fim $A^{+}$ strains PC31 and DSM 6601 proved to provide a better background for presentation of chimeric FimA proteins than did the MS7 strain $(\Delta f i m)$. This could be due to coassembly of wild-type and chimeric FimA proteins into fimbrial organelles. However, a positive influence of a chromosomally located wild-type fim gene cluster on the plasmid-encoded fim genes was apparently not the only criterion for a better display, since when comparing PC31 and DSM 6601, both of which have a resident 

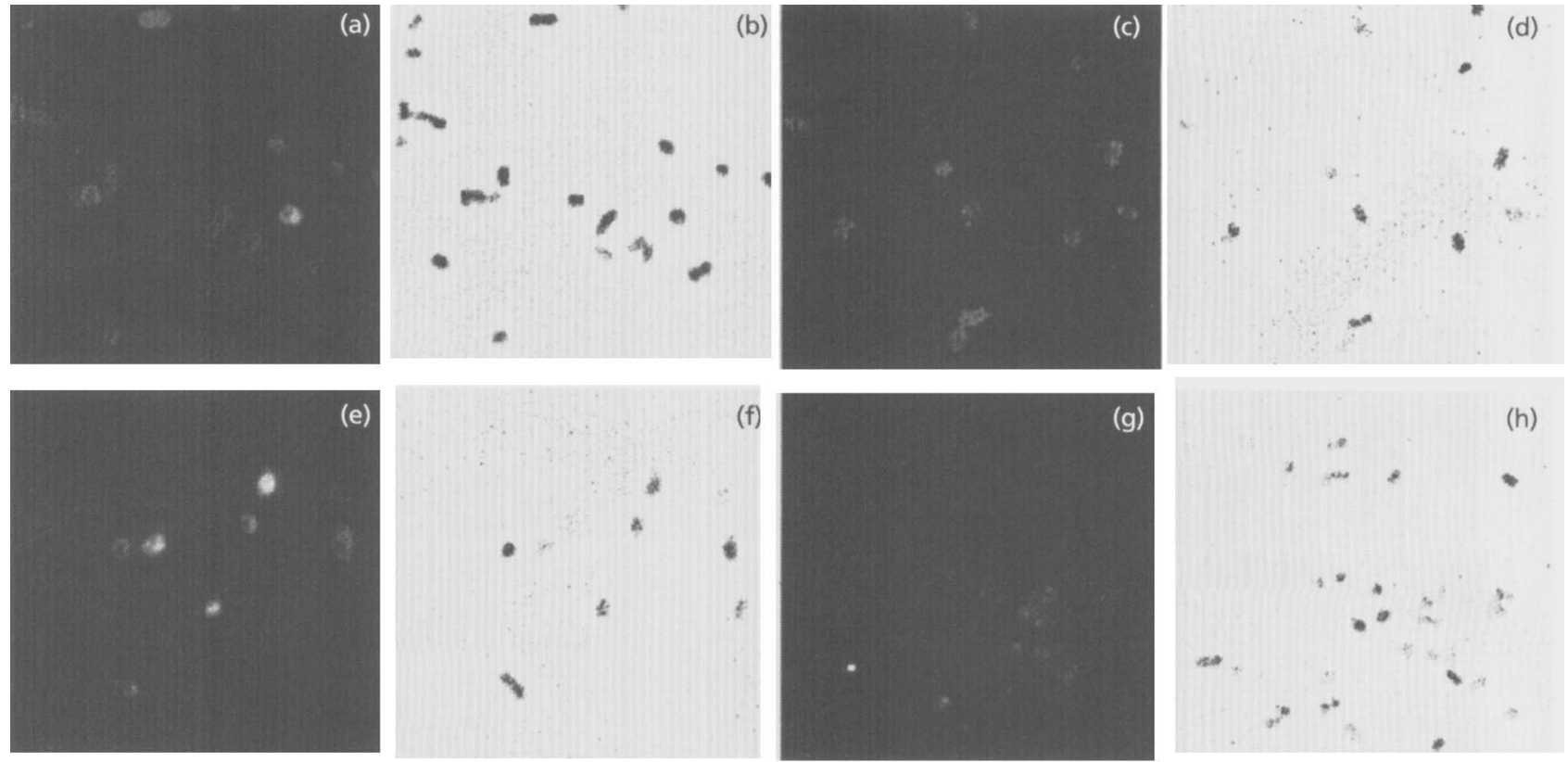

(i)
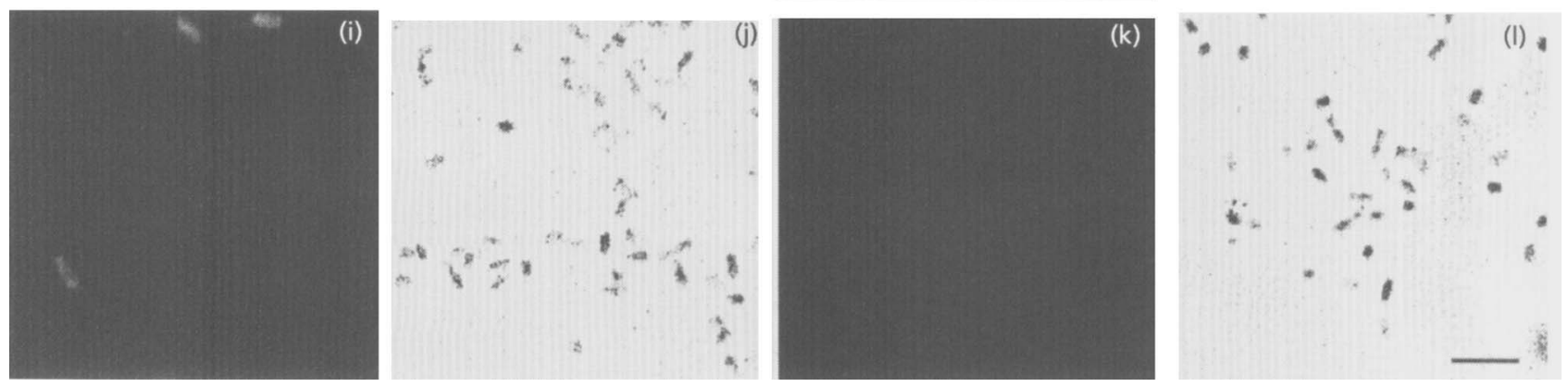

Fig. 5. Surface display of chimeric fimbriae assessed by fluorescence microscopy (FM) and DICM of $E$. coli DSM 6601 cells. In all cases fluorescence-labelled secondary antisera were used, the type of primary serum employed is indicated. Bacteria were fixed, reacted with primary antiserum, washed, reacted with FITC-labelled secondary antiserum, washed and subjected to microscopy. Images were recorded with CCD technology (see Methods). (a) FM and (b) DICM of cells harbouring plasmid pBSO5 (encoding a fimA gene with a single cholera insert) reacted with anti-type 1 serum (primary serum). (c) FM and (d) DICM of cells harbouring plasmid PBSO5 (encoding a fimA gene with a single cholera insert) reacted with anti-CTB serum (primary serum). (e) FM and (f) DICM of cells harbouring plasmid pBSO4 (encoding a fimA gene with a tandem cholera insert) reacted with anti-type 1 serum (primary serum). (g) FM and (h) DICM of cells harbouring plasmid pBSO4 (encoding a fimA gene with a tandem cholera insert) reacted with anti-CTB serum (primary serum). (i) FM and (j) DICM of cells harbouring plasmid pLBJ312 (fimA) reacted with anti-type 1 serum (primary serum). (k) FM and (I) DICM of cells harbouring plasmid pLBJ312 (fimA) reacted with anti-CTB serum (primary serum). Bar, $10 \mu \mathrm{m}$.

wild-type fim gene cluster, the latter was clearly a better presenter of engineered versions of FimA. A multitude of factors, such as higher levels of chaperones and members of the general secretory pathway and lower levels of scavenging proteinases, might cause the DSM 6601 strain to be such an amenable host in this respect.

We believe that the fimbrial presentation system has a considerable potential, firstly because of the antigenamplification effect inherent in the fact that the major structural protein, and thereby the heterologous sequence, is present in large amounts predominantly on the bacterial surface (up to 500000 copies per cell in the wild-type situation). Surface presentation seems to be important for a strong immune-response in the context of live bacterial vaccines, e.g. in attenuated Salmonella strains it has been shown that a stronger immune response was elicited when the heterologous antigen was exposed on the bacterial surface (Hormaeche et al., 1992; Leclerc et al., 1991). Secondly, fimbriae are easy to purify, a fact which might be relevant for a component vaccine approach.

The present system is an example of a strategy that has been pursued by several investigators in recent years, namely to use naturally occurring bacterial surface organelles for presentation of foreign epitopes. Two major approaches, employing fimbriae and flagella, respectively, have been used to this end (Newton et al., 1989; Pallesen \& Klemm, 1994; Wu et al., 1989). Previous studies employing the major subunit protein of various fimbriae have succeeded in displaying hetero- 

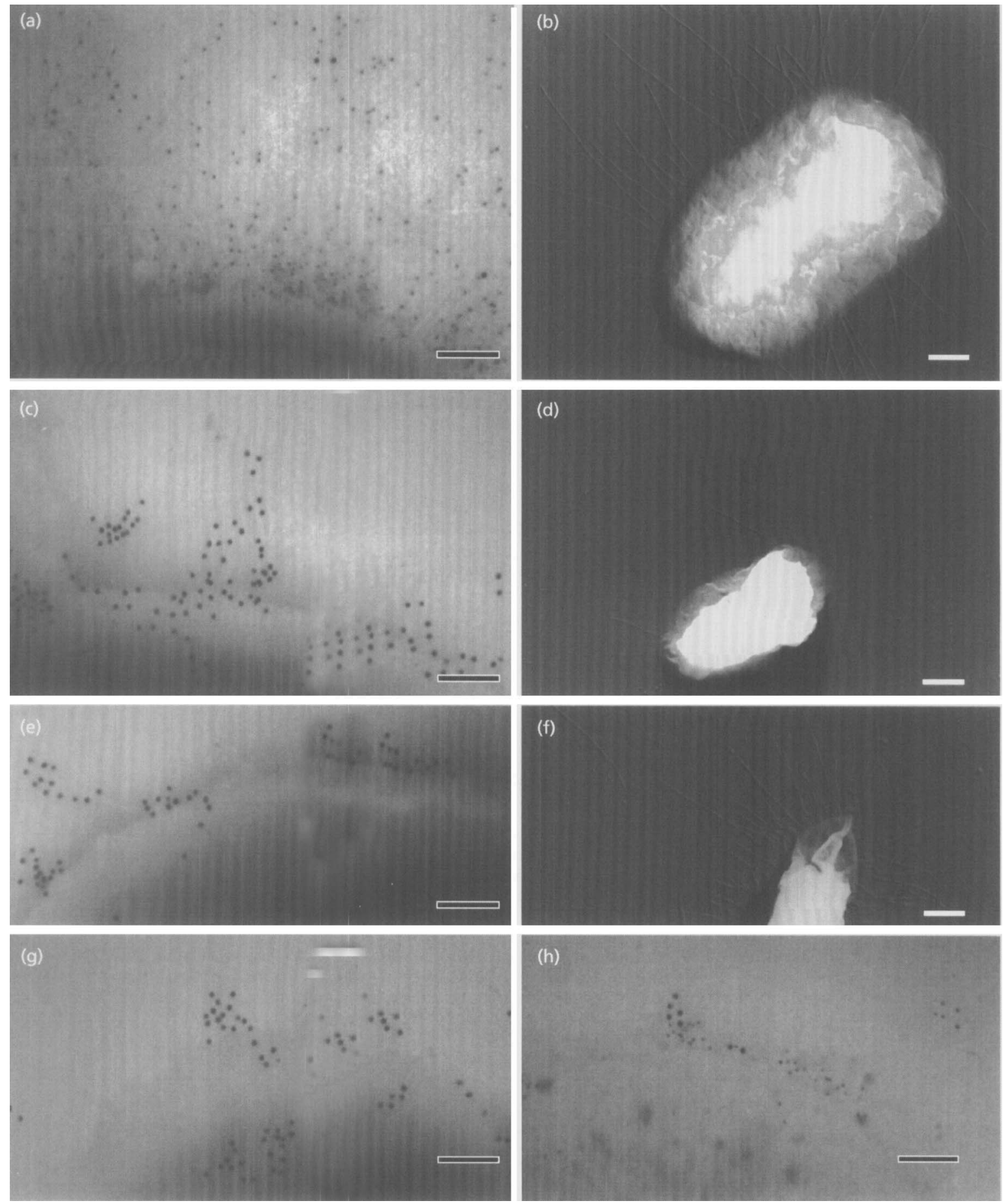

(h)

Fig. 6. Double-labelled immunoelectron micrographs (a, $c, e, g$ and $h)$, showing labelling of FimA $(5 \mathrm{~nm})$ and the CTB segment on chimeric FimA $(10 \mathrm{~nm})$. Bacteria were adsorbed to the surface of the grid and incubated with a mixture of anti-CTB rabbit serum $(1: 100)$ and anti-FimA monoclonal antibody (1:5). As secondary antibodies a mixture of goat antimouse serum conjugated with $5 \mathrm{~nm}$ colloidal gold particles and goat anti-rabbit serum conjugated with $10 \mathrm{~nm}$ colloidal gold particles were used in dilution $1: 20$. Surface structures of the bacteria were analysed by shadow casting with tungsten at an angle of $30^{\circ}$ (b, d and f). (a, b) pLBJ318/MS7; (c, d), pBSO5/MS7; (e, f) pBSO5/DSM 6601; (g) pBSO5/PC31; (h) pBSO1/MS7. Black bars (a, c, e, g and h), $100 \mathrm{~nm}$. White bars (b, $d$ and f), $200 \mathrm{~nm}$.

logous segments of sizes up to, but not beyond, 20 amino acid residues (for review see Pallesen \& Klemm, 1994). In the present study we have succeeded in the authentic display of segments of 34 foreign amino acid residues, a reasonable improvement. We have recently studied the potential of the FimH minor component of type 1 
(a)

(b)

СTB ch.f. wt.f. CTB ch.f. wt.f. CTB ch.f. wt.f.

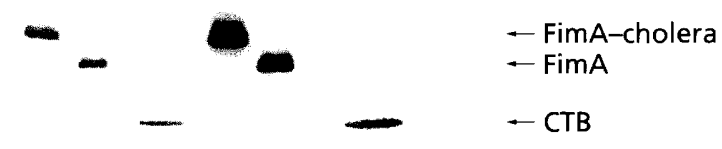

Fig. 7. Immunoblots showing the immune response of rabbits immunized with (a) wild-type FimA (serum dilution 1:1000), (b) chimeric FimA harbouring a CTB-segment in position 25 (serum dilution $1: 250$ ) and (c) CTB (serum dilution 1:40000). Three identical runs of $C T B$, chimeric FimA and wild-type FimA are shown in (a), (b) and (c).

fimbriae for display of heterologous sequences (Pallesen et al., 1995), and have, in this background succeeded in displaying even larger segments (58 amino acids). It might be that the FimH protein is able to accept larger heterologous grafts than FimA. However, one drawback of $\mathrm{FimH}$ is the lower ratio of this protein when integrated into the fimbriae; about a hundredfold less than FimA. The high valency display format provided by the FimA presentation system may be a significant advantage in vaccine design.

Future research will reveal whether a new generation of vaccines can be based on chimeric fimbriae or flagella, which fulfil the requirements for successful exposure and antigenicity of the heterologous epitopes while at the same time maintaining the functionality of the parental proteins.

\section{ACKNOWLEDGEMENTS}

The authors wish to thank Pernille Olsson for expert technical assistance. E. coli strain MS7 was generously made by Mark Schembri. This work was supported by The Danish Medical and Technical Research Councils and the WHO/UNDP Programme for Vaccine Development (ref. no. MIM/I5/ $181 / 171)$.

\section{REFERENCES}

Birnboim, H. C. \& Doly, J. (1979). A rapid alkaline extraction procedure for screening recombinant plasmid DNA. Nucleic Acid Res 7, 1513-1523.

Blum, G., Marre, R. \& Hacker, J. (1995). Properties of Escherichia coli strains of serotype O6. Infection 23, 234-236.

Brinton, C. C., Jr (1965). The structure, function, synthesis and genetic control of bacterial pili and a molecular model for DNA and RNA transport in Gram negative bacteria. Trans NY Acad Sci 27, 1003-1054.

Dodd, D. C. \& Eisenstein, B. I. (1984). Dependence of secretion and assembly of type 1 fimbrial subunits on normal protein export. J Bacteriol 159, 1077-1079.
Duguid, J. P. \& Old, D. C. (1980). Adhesive properties of Enterobacteriaceae. In Receptors and Recognition, series B, vol. 6, pp. 186-217. Edited by E. Beachey. London: Chapman \& Hall.

Hedegaard, L. \& Klemm, P. (1989). Type 1 fimbriae of Escherichia coli as carriers of heterologous antigenic sequences. Gene $\mathbf{8 5}$, 115-124.

Hormaeche, C. E., Dougan, G. \& Chatfield, S. N. (1992). Live attenuated salmonella: oral vaccines for salmonellosis and combined vaccines carrying heterologous antigens. In The Release of Genetically Modified Microorganisms, pp. 71-83. Edited by D. E. S. Stewart-Tull \& M. Sussmann. New York: Plenum Press.

Hultgren, S. J., Abraham, S. N., Caparon, M., Falk, P., St Geme, J. W., III \& Normark, S. (1993). Pilus and non pilus bacterial adhesins: assembly and function in cell recognition. Cell 73, 887-901.

Jacob, O. C., Sela, M. \& Arnon, R. (1983). Antibodies against synthetic peptides of the $B$ subunit of cholera toxin: crossreaction and neutralization of the toxin. Proc Natl Acad Sci USA 80, 7611-7615.

Jones, C. H., Pinkner, J. S., Nicholes, A. V., Slonim, L. N., Abraham, S. N. \& Hultgren, S. J. (1993). FimC is periplasmic PapD-like chaperone that directs assembly of type 1 pili in bacteria. Proc Natl Acad Sci USA 90, 8397-8401.

Klemm, P. (1984). The fimA gene encoding the type 1 fimbrial subunit of Escherichia coli, nucleotide sequence and primary structure of the protein. Eur J Biochem 143, 395-399.

Klemm, P. (1992). FimC, a chaperone-like periplasmic protein of Escherichia coli involved in biogenesis of type 1 fimbriae. Res Microbiol 143, 831-838.

Klemm, P. \& Christiansen, G. (1987). Three fim genes required for the regulation of length and mediation of adhesion of Escherichia coli type 1 fimbriae. Mol Gen Genet 208, 439-445.

Klemm, P. \& Christiansen, G. (1990). The fimD gene required for cell surface localization of Escherichia coli type 1 fimbriae. Mol Gen Genet 220, 334-338.

Klemm, P. \& Krogfelt, K. A. (1994). Type 1 fimbriae of Escherichia coli. In Fimbriae, Adhesion, Genetics, Biogenesis and Vaccines, pp. 9-28. Edited by P. Klemm. Boca Raton, FL: CRC Press.

Klemm, P., Jørgensen, B. J., van Die, I., de Ree, H. \& Bergmans, H. (1985). The fim genes responsible for synthesis of type 1 fimbriae in Escherichia coli. Mol Gen Genet 199, 410-414.

Klemm, P., Christiansen, G., Kreft, B., Marre, R. \& Bergmans, H. (1994). Reciprocal exchange of minor components of type 1 and F1C fimbriae results in hybrid organelles with changed receptor specificity. J Bacteriol 176, 2227-2234.

Krogfelt, K. A. (1991). Bacterial adhesion: genetics, biogenesis, and role in pathogenesis of fimbrial adhesins of Escherichia coli. Rev Infect Dis 13, 721-735.

Krogfelt, K. A. \& Klemm, P. (1988). Investigation of minor components of Escherichia coli type 1 fimbriae: protein chemical and immunological aspects. Microb Pathog 4, 231-238.

Krogfelt, K. A., Bergmans, H. \& Klemm, P. (1990). Direct evidence that the FimH protein is the mannose specific adhesin of Escherichia coli type 1 fimbriae. Infect Immun 58, 1995-1998.

Leclerc, C., Charbit, A., Martineau, P., Deriaud, E. \& Hofnung, M. (1991). The cellular location of a foreign $B$ cell epitope expressed by recombinant bacteria determines its $\mathrm{T}$ cell-dependent characteristics. J Immunol 147, 3545-3550.

Levine, M. M., Giron, J. \& Noriega, F. (1994). Fimbrial vaccines. In Fimbriae, Adhesion, Genetics, Biogenesis and Vaccines, pp. 271-286. Edited by P. Klemm. Boca Raton, FL: CRC Press. 
McMichael, J. C. \& Ou, J. T. (1979). Structure of common pili from Escherichia coli. J Bacteriol 138, 969-975.

Merrit, E. A., Sarfaty, S., van den Akker, F., L'Hoir, C. E., Martial, J. A. \& Hol, W. G. J. (1994). Crystal structure of cholera toxin Bpentamer bound to receptor $\mathrm{G}_{\mathrm{MI}}$ pentasaccharide. Protein Sci 3, 166-175.

Moon, H.W. \& Bunn, T. O. (1993). Vaccines for preventing enterotoxigenic Escherichia coli infections in farm animals. Vaccine 11, 213-219.

Newton, S. M. C., Jacob, C. O. \& Stocker, B. A. D. (1989). Immune response to cholera toxin epitope inserted into Salmonella flagellin. Science 244, 70-72.

Pallesen, L. \& Klemm, P. (1994). Chimeric fimbrial vaccines. In Fimbriae, Adhesion, Genetics, Biogenesis and Vaccines, pp. 289-294. Edited by P. Klemm. Boca Raton, FL: CRC Press.

Pallesen, L., Poulsen, L. K., Christiansen, G. \& Klemm, P. (1995). Chimeric FimH adhesin of type 1 fimbriae: a bacterial display system for heterologous sequences. Microbiology 141, 2839-2848.

Pugsley, A. P. (1993). The complete secretory pathway in Gramnegative bacteria. Microbiol Rev 57, 50-108.

Russell, P. V. \& Orndorff, P. E. (1992). Lesions in two Escherichia coli type 1 pilus genes alter pilus number and length without affecting receptor binding. J Bacteriol 174, 5923-5935.

Sanger, F., Nicklen, S. \& Coulson, A. R. (1977). DNA sequencing with chain-terminating inhibitors. Proc Natl Acad Sci USA 74, 5463-5467.

Schembri, M., Pallesen, L., Connell, H. \& Klemm, P. (1996). Linker insertion mutagenesis of the FimH adhesin of type 1 fimbriae in an Escherichia coli fimH-null host. FEMS Microbiol Lett 137, 257-263.

Shoham, M., Scherf, T., Anglister, J., Levitt, M., Merrit, E. A. \& Hol, W. G. J. (1995). Structural diversity in a conserved cholera toxin epitope involved in ganglioside binding. Protein Sci 4, 841-848.

Wu, J. Y., Newton, S., Judd, A., Stocker, B. \& Robinson, W. S. (1989). Expression of immunogenic epitopes of hepatitis B surface antigen with hybrid flagellin proteins by a vaccine strain of Salmonella. Proc Natl Acad Sci USA 86, 4726-4730.

Received 26 November 1996; revised 30 January 1997; accepted 3 February 1997. 